\title{
VECTORIAL WAVEGUIDE REFLECTOMETER FOR DIELECTRIC CHARACTERIZATION OF MATERIALS UNDER POWER MICROWAVES
}

\author{
Jordi J. Mallorquí (*), Albert Aguasca, Serni Ribó \\ Electromagnetic and Photonics Engineering Group (EEF) \\ Dept. of Signal Theory and Communications, Universitat Politècnica de Catalunya \\ D3-Campus Nord-UPC, C/ Jordi Girona 1-3, 08034 Barcelona (Spain) \\ E-Mail: mallorqu@voltor.upc.es; Tel: 34-93 401 72 29; Fax: 34-93 4017232
}

Introduction

The temperature rise in a material modifies its physical properties, particularly its dielectric permittivity. In many applications involving relatively high levels of power the electrical behavior of the different materials will change as they are heated by the radiation. For instance, the numerical codes that simulates the behavior of microwave heating processes in order to improve the design of the feeding antennas must take into account the load variations with temperature [1]. The measurement of the changing dielectric characteristics of materials is of great interest for the industry. Previous works used completelly filled waveguides near a shorting plate [2]. The method supplied excellent results at low power levels, while the sample was not heated. When the power is risen, the field distribution of the $\mathrm{TE}_{10}$ mode causes a non-uniform heating of the sample and the measured permittivity corresponds to an average value. In order to reduce this problem a method using a partially filled waveguide is presented. The reduced sample dimensions and its positioning into the waveguide assures a near homogeneous power distribution implying a uniform heating.

\section{The Algorithm}

In partially filled waveguides with the material interference perpendicular to the $x$ or $y$ axis the traditional $\mathrm{TE}^{2}$ and $\mathrm{TM}^{2}$ modes cannot satisfy the boundary conditions of the structure. It can be demonstrated that the field configurations that are combinations of $\mathrm{TE}^{2}$ and $\mathrm{TM}^{2}$ modes can be solutions and satisfy the boundary conditions of such partially filled waveguides. The modes are referred to as hybrid modes or longitudinal section electric (LSE) or longitudinal section magnetic (LSM) modes [3]. Making use of the transverse resonance method (TRM), the cross section of the waveguide is represented as a transmission line system. The fields must satisfy the tranverse wave equation (1), and the resonances of this transverse network will yield expressions for the propagation constants of the waveguide, $\gamma$. Then in each homogeneous section 


$$
\begin{gathered}
\gamma^{2}=k_{x 1}^{2}+k_{y 1}^{2}-k_{0}^{2}=k_{x 1}^{2}-k_{0}^{2} \\
\gamma^{2}=k_{x 2}^{2}+k_{y 2}^{2}-\varepsilon_{r} k_{0}^{2}=k_{x 2}^{2}-\varepsilon_{r} k_{0}^{2}
\end{gathered}
$$

where $\mathrm{k}_{\mathrm{x}}$ is the propagation constant component in the $\mathrm{x}$ direction. As the waveguide operates in the $\mathrm{TE}_{10}$ mode, $\mathrm{k}_{\mathrm{y}}=0$. The subindex 1 refers to the empty part of the guide and the subindex 2 to the filled one. An equivalent transmission line model of the transversal section allows obtain equations (2) and (3)

$$
\begin{gathered}
Z_{1}=\frac{j \omega \mu}{k_{x 1}} \tan \left(k_{x 1} d\right) \\
Z_{2}=\frac{\omega \mu}{k_{x 2}} \frac{k_{x 1}^{-1} \tan \left(k_{x 1} d\right)+j k_{x 2}^{-1} \tan \left(k_{x 2} e / 2\right)}{k_{x 2}^{-1}-k_{x 1}^{-1} \tan \left(k_{x 1} d\right) \tan \left(k_{x 2} e / 2\right)}
\end{gathered}
$$

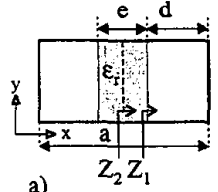

a)
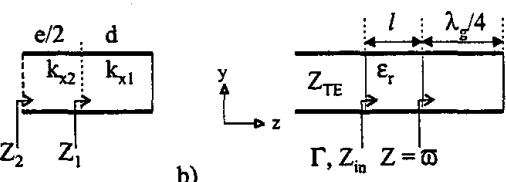

Fig. 1 Transversal (a) and longitudinal (b) transmission line models of the partially filled waveguide.

Assuming a symmetrical field distribution excited by a $\mathrm{TE}_{10}$ mode, there is a field maximum in the center of the waveguide. Then $Z_{2}$ must be infinite if the material had no losses. In a more general case, with lossy materials $Z_{2}$ should present a maximum. For a given geometry and material, the inverse transcendental equation (3), combined with (1), can be minimized in order to get the propagation constant, $\gamma$

The value of the input impedance at the reference plane, $Z_{\text {in }}$, can be obtained with a transmission line model as it can be seen in Fig. 1b

$$
Z_{\text {in }}=\frac{\omega \mu}{\gamma} \tan (j \gamma \ell-\pi / 2)
$$

whith $l$ being the length of the partially filled waveguide when loaded with a short circuit at a distance of $\lambda_{g} / 4$. The reflection coefficient, referred to the empty waveguide impedance, can also be computed

$$
Z_{\text {in }}=Z_{T E} \frac{1+\Gamma}{1-\Gamma}
$$


As the reflection coefficient from a known material and dimensions inside the waveguide can be found, an "inverse" procedure can be applied in order to get the unknown dielectric constant from a measured reflection coefficient. The sequence is the following: first the input impedance at the reference plane is computed from the measured reflection coefficient. Second, the propagation constant in the filled waveguide is obtained from the impedance. Third and final, the unknown permittivity is obtained from the complex propagation constant. The steps two and three are solved minimizing the functions obtained from the previous transcendent equations [4]

\section{Experimental set-up}

Fig. 2 shows the experimental set-up used to measure the dielectric properties of the different materials.

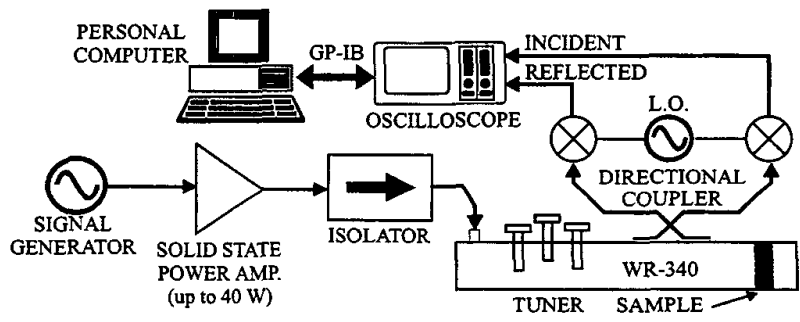

Fig. 2 Experimental set-up for dielectric measurements.

A three-screw tuner, placed between the isolator and the directional couplers, is used to match the sample and maximize the power transfer from the amplifier. The calibration of the measurement system is carried out with a set of four displaced short circuits, which gives better results than the traditional set of matched load, short and open circuits.

Results

Fig. 3-a shows the measured permittivity of a teflon sample of 10 by $10 \mathrm{~mm}$ used to validate the behavior of the measurement system. The incident power is $1 \mathrm{~W}$ and the real part of the permittivity, around 2.1 , does not change with time. The measured real part agrees with the values found in the literature [5]. Fig. 3$b$ presents the permittivity variation of a rubber sample of $19 \times 10 \mathrm{~mm}$ when the incident power is $10 \mathrm{~W}$. The initial temperature of the sample was $5^{\circ} \mathrm{C}$ and at the end of the heating process $70^{\circ} \mathrm{C}$. As it can be seen, both the real and imaginary parts of permittivity change as the temperature rises. A new permittivity value is obtained every two seconds. 


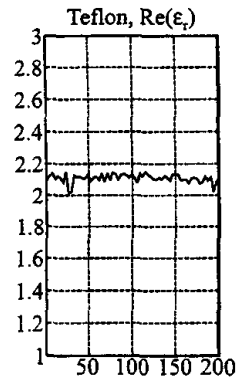

a)

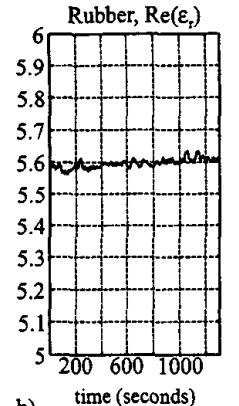

b)

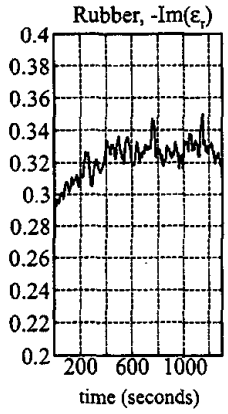

time (seconds)

Fig. 3. Dielectric measurements: (a) teflon sample, (b) rubber sample.

The problem of multiple equivalent solutions is overcomed by the a-priori information of the material under test.

Conclusions

A measurement system able to characterize the dynamic behavior of materials as they absorb RF power has been presented. The small dimensions of the sample and its placement where the field has its maximum assures a uniform heating. Then the system is able to measure the correct permittivity values changing with temperature. The measurement system will be combined with a small fiber optic temperature probe placed inside the sample that will allow complement the permittivity values with the sample temperature.

Acknowledgements

This work has been sponsored by the company CIM-D'OR and the Spanish Government under grant PETRI 95-0084-OP.

\section{References}

[1] F. Torres, B. Jecko, "Complete FDTD Analysis of Microwave Heating Processes in Frequency-Dependent and Temperature-Dependent Media", IEEE Trans. on Microwave Theory and Techniques, Vol. 45, No. 1, pp.108-177, January 1997.

[2] S. I. Ganchev, J. Bhattacharyya, et al., "Microwave Diagnosis of Rubber Compounds", IEEE Trans. on Microwave Theory and Techniques, Vol. 42, No. 1, pp. 18-24, January 1994.

[3] C. A. Balanis, "Advanced Engineering Electromagnetics", John Wiley \& Sons, 1989.

[4] "MATLAB Optimization Toolbox", The MathWorks Inc., 1994.

[5] A..R. Von Hippel, "Dielectric Materials and Applications", The MTT Press, 1954. 